\title{
e-ISSN $1983-0572$ \\ Riqueza de Borboletas (Lepidoptera) em um Fragmento Urbano de Cerrado em Mato Grosso do Sul, Brasil
}

\author{
Paulo Alexandre Bogiani ${ }^{1}$, Rodrigo Aranda1 $\&$ Camila de Oliveira Freitas Machado²
}

1. Universidade Federal de Mato Grosso do Sul, e-mail: pbogiani@yahoo.com.br (Autor para correspondência ${ }^{\bowtie}$ ), rodrigoaranda.biologo@gmail.com. 2. Universidade de São Paulo, e-mail: mila ofm@hotmail.com.

EntomoBrasilis 5 (2): 93-98 (2012)

\begin{abstract}
Resumo. Borboletas estão entre os artrópodes melhor estudados, pois participam de diversas interações ecológicas. Apesar de já existirem muitos inventários de borboletas publicados no Brasil, nenhuma informação ainda existe em Mato Grosso do Sul. O presente estudo teve como objetivo avaliar a riqueza de espécies de borboletas encontradas em um remanescente urbano de cerrado em Campo Grande, Centro-Oeste do Brasil, além de indicar a presença de espécies raras e indicadora de ambiente preservado. Foram registradas 62 espécies de borboletas, pertencentes a seis famílias e 15 subfamílias. Entre as espécies registradas, 28 pertencem à família Nymphalidae (45,1\%), 16 a Hesperiidae (25,8\%), oito a Lycaenidae (12,9\%), seis a Pieridae (9,7\%) e quatro para Riodinidae (6,5\%). As estimativas de riqueza Jack-Knife (de $1^{\text {a }}$ ordem) e Chao2 mostram que entre 81 e $86 \%$ das espécies, respectivamente, foram registradas durante o período do estudo para a área. A lepidopterofauna encontrada não apresenta espécies cuja presença indica ambiente preservado. Embora a área de estudo seja pequena, estando imersa em uma matriz urbana e sendo constantemente impactada, a reserva representa um ambiente importante para a conservação de borboletas na cidade de Campo Grande.
\end{abstract}

Palavras-Chave: Espécies raras; Levantamento; Remanescente urbano de cerrado; Reserva Particular de Patrimônio Natural; Riqueza de espécies.

\section{Butterflies Species (Lepidoptera) in an Urban fragment of Savannah in Mato Grosso do Sul, Brazil.}

Abstract. Butterflies are among the better studied arthropods, because they participate in several ecological interactions. In spite of already many inventories of butterflies have been published in Brazil, still any information exist in Mato Grosso do Sul. The present study aimed to evaluate the species richness of butterflies found in an urban remnant of savannah in Campo Grande, Midwest of Brazil, besides indicating the presence of rare species and indicative of preserved atmosphere. We registered 62 species of butterflies, ranging in six families and 15 subfamilies, for the area. Among the registered species, 28 belong to the family Nymphalidae (45.1\%), 16 to Hesperiidae (25.8\%), 8 to Lycaenidae (12.9\%), 6 to Pieridae (9.7\%) and 4 to Riodinidae (6.5\%). The wealth estimates Jack-Knife (1st order) and Chao2 show that among 81 and $86 \%$ of species, respectively, were registered during the period of the study for the area. The found lepidopterofauna didn't present species whose presence indicates preserved atmosphere. Although the small study area, being immersed in an urban matrix and constantly being impacted, the reserve represents an important environment for the conservation of butterflies in the city of Campo Grande.

Keywords: Check list; Private Reserve of Natural Heritage; Rare species; Remainder urban of Savannah; Richness of species.

$\mathbf{N}$ avaliação e monitoramento da perda de biodiversidade é imprescindível o estudo da riqueza de espécies e o desenvolvimento de diferentes tipos de pesquisas envolvendo vegetais e animais (Wood \& GILLMAn 1998). As modificações humanas na paisagem e o processo de urbanização têm levado à destruição, fragmentação e ao isolamento de habitats naturais, com consequente prejuízo para a biodiversidade aumentado assim, a importância das áreas remanescentes como refúgio para a fauna e flora (FAHRIG 2003; Silva et al. 2007). Os efeitos advindos das modificações, geralmente promovem a interrupção do fluxo gênico, comprometendo a reprodução e manutenção das espécies (WILSON 1997). Por isso o interesse na conservação do ecossistema vem crescendo (DAILY \& EHRLicH 1995; Hughes et al. 2000) e vários cientistas propõem a utilização de táxons conhecidos para estudos da conservação (IsERHARD \& RoMANOWSKI 2004).

De modo geral, Lepidoptera têm sido sugeridos como bons indicadores biológicos, pois possuem ciclos reprodutivos dinâmicos, respondendo assim, rapidamente às mudanças na vegetação e no clima, são conspícuas e por isso fáceis de serem observadas e amostradas em qualquer época do ano, além de terem taxonomia bem conhecida (BRown 1991; FreITAs et al. 2003). Estão envolvidas em muitas interações no meio em que vivem, como por exemplo, polinização e predação. Servem de modelo em pesquisas de ecologia de populações e comportamento (DessuY \& Morais 2007).

Segundo Ruszczyк (1999) a urbanização interfere no número de borboletas de uma dada área, sendo que quanto maior o grau de urbanização, menor será o número de espécies de borboletas, sugerindo a existência de espécies sensíveis à urbanização e seu potencial como indicadores biológicos. Santos (2004), diz que sem um conhecimento mínimo sobre organismos que ocorrem em cada local, é virtualmente impossível desenvolver qualquer projeto de conservação. Em relação aos remanescentes de vegetação, trabalhos de inventários sobre as comunidades de borboletas indicam a importância e efetividade desses habitats para manutenção da fauna regional (BRown JR. \& FreITAS 2000; CoLLIER etal. 2006). Esses remanescentes são refúgiosimportantes tanto para plantas quanto para animais não adaptados a matriz urbana (RoDRIGues et al. 1993). Para as borboletas, esses habitats garantem a manutenção de uma alta riqueza de espécies nas cidades (Fortunato \& RuszczYK 1997). 
No domínio do cerrado ocorre aproximadamente um terço da biota brasileira, com riqueza mínima estimada de 320.000 espécies (DiAs 1996), sendo que os insetos compreendem a maior parte das espécies da fauna (Diniz \& Morais 1995). Lepidoptera corresponde a segunda maior ordem da classe Insecta (FonsECA et al. 2006) com uma riqueza de aproximadamente 146.000 espécies descritas, entre borboletas e mariposas. No Brasil, devem existir cerca de 25.000 espécies de borboletas (HEPPNER 1991; Beccaloni \& Gaston 1995; Brown Jr. \& Freitas 1999). Apesar da elevada riqueza de espécies de borboletas na região Neotropical, o número de espécies ou subespécies endêmicas no cerrado é pequeno (<6\%) (EMERY et al. 2006).

No Brasil existem vários estudos envolvendo a fauna de borboletas, principalmente nas regiões de Mata Atlântica (BRown \& Freitas 2000) e de cerrado (CAMARgo \& BeCKER 1999; EMERY et al. 2006). Sabe-se ainda que existem algumas áreas com alta riqueza de espécies que não apresentam estudos ou estes são muito escassos (BECCALONI \& GASTON 1995). O primeiro estudo aprofundado sobre a fauna de borboletas do cerrado foi realizado por Brown \& Mielke (1967a, b) que apresentam listagem com aproximadamente 700 espécies de borboletas para toda região do cerrado. O estado do Mato Grosso do Sul, está entre os estados onde são escassas as informações sobre lepidopterofauna. AOKI \& SIGRIST (2006), amostrando visitantes florais na região leste do estado, registraram 43 espécies de quatro famílias de borboletas.

Este trabalho teve como objetivo avaliar a riqueza de espécies de borboletas localizadas na Reserva Particular de Patrimônio Natural, no campus da Universidade Federal de Mato Grosso do Sul, Campo Grande, Mato Grosso do Sul. Pretendeu-se reunir informações sobre registros de borboletas para o estado, para que posteriormente possam servir de base para novos estudos sobre a conservação da área e indicar a presença de espécies raras e/ou indicadoras de ambiente preservado, além de contribuir para o conhecimento da lepidopterofauna da região.

\section{MATERIAL E MÉTODOS}

Área de Estudo. Este estudo foi realizado de agosto de 2008 a julho de 2009, no município de Campo Grande, Mato Grosso do Sul, em duas áreas: i) um remanescente contíguo de cerrado com 36,5 ha, que apresenta vegetação constituída de cerrado e cerradão (Munin et al. 2008) e ii) um trecho de mata ciliar do córrego Bandeiras que corta a Universidade Federal de Mato Grosso do Sul (UFMS) com 24 ha e apresenta uma vegetação densa, semelhante às matas de galeria e apresentando alagamento na época das chuvas (Figura 1). As duas áreas $\left(20^{\circ} 27^{\prime} \mathrm{S}\right.$ e $54^{\circ} 37^{\prime} \mathrm{W}$, 530 m de altitude) pertencem à Reserva Particular de Patrimônio Natural da UFMS (RPPN/UFMS). A região apresenta clima do tipo Tropical Chuvoso de Savana (subtipo Aw) (KöPPEN 1948) com inverno seco e frio (maio a agosto) e verão chuvoso e quente (dezembro a março). A precipitação média anual é de $1.532 \mathrm{~mm}$, a umidade relativa é geralmente baixa, raramente atingindo $80 \%$ e as temperaturas médias anuais variam entre 20 e $22{ }^{\circ} \mathrm{C}$ (EMBRAPA - CNPGC 1985). A reserva sofre interferência antrópica direta, pois é aberta para visitas além das atividades acadêmicas e de pesquisa e também faz divisa com algumas ruas e uma grande avenida de onde recebe lixo, além de sofrer frequentes perturbações com a ida de caminhões para ali jogarem entulhos.

Amostragem. As coletas foram feitas mensalmente, com auxílio de rede entomológica tipo puçá, armadilhas iscadas para coleta de borboletas (modelo baseado em Almeida et al. 2003) e coleta em plantas cujas flores são visitadas por diversos tipos de insetos ou com síndrome de psicofilia, sendo o trabalho de campo realizado geralmente em três etapas:

i) primeira etapa: foram realizadas coletas com puçás entre 10 e $14 \mathrm{~h}$, por no mínimo duas pessoas que caminharam de forma aleatória pelas áreas de estudo. ii) segunda etapa: foram utilizadas 15 armadilhas, dispostas de forma aleatória nas áreas de estudo (sendo que 10 armadilhas no remanescente de cerrado e 5 na mata ciliar). Nas armadilhas foram utilizadas como iscas: fezes (de capivara), saliva humana, carne em decomposição, banana amassada com garapa e urina humana (FREITAS et al. 2003). As armadilhas foram colocadas entre $7 \mathrm{e}$ 9h e vistoriadas às $12 \mathrm{e} 15 \mathrm{~h}$ sendo que na última vistoria as armadilhas eram retiradas.

iii) terceira etapa: foram feitas observações em flores entre 10 e $14 \mathrm{~h}$ totalizando 30 horas de observação em indivíduos com flores das famílias Asteraceae e Verbenaceae, que são famílias que possuem flores psicófilas. Os espécimes foram acondicionados em envelopes entomológicos e rotulados. Foram ainda devidamente preparados, sendo posteriormente separados em nível de família, procedendo-se a identificação em nível de espécie por especialistas na área, sendo estes, depositados no Museu de Zoologia do Instituto de Biologia da Universidade Estadual de Campinas.

Para cálculo da riqueza de espécies foram utilizados os índices Chao 2 e Jack-knife 1 ordem, calculados com o programa BioDiversity Pro (McAleceE et al. 1997). A determinação de espécies raras e/ou indicadoras de ambientes preservados foi baseada em Brown \& Freitas (2000).
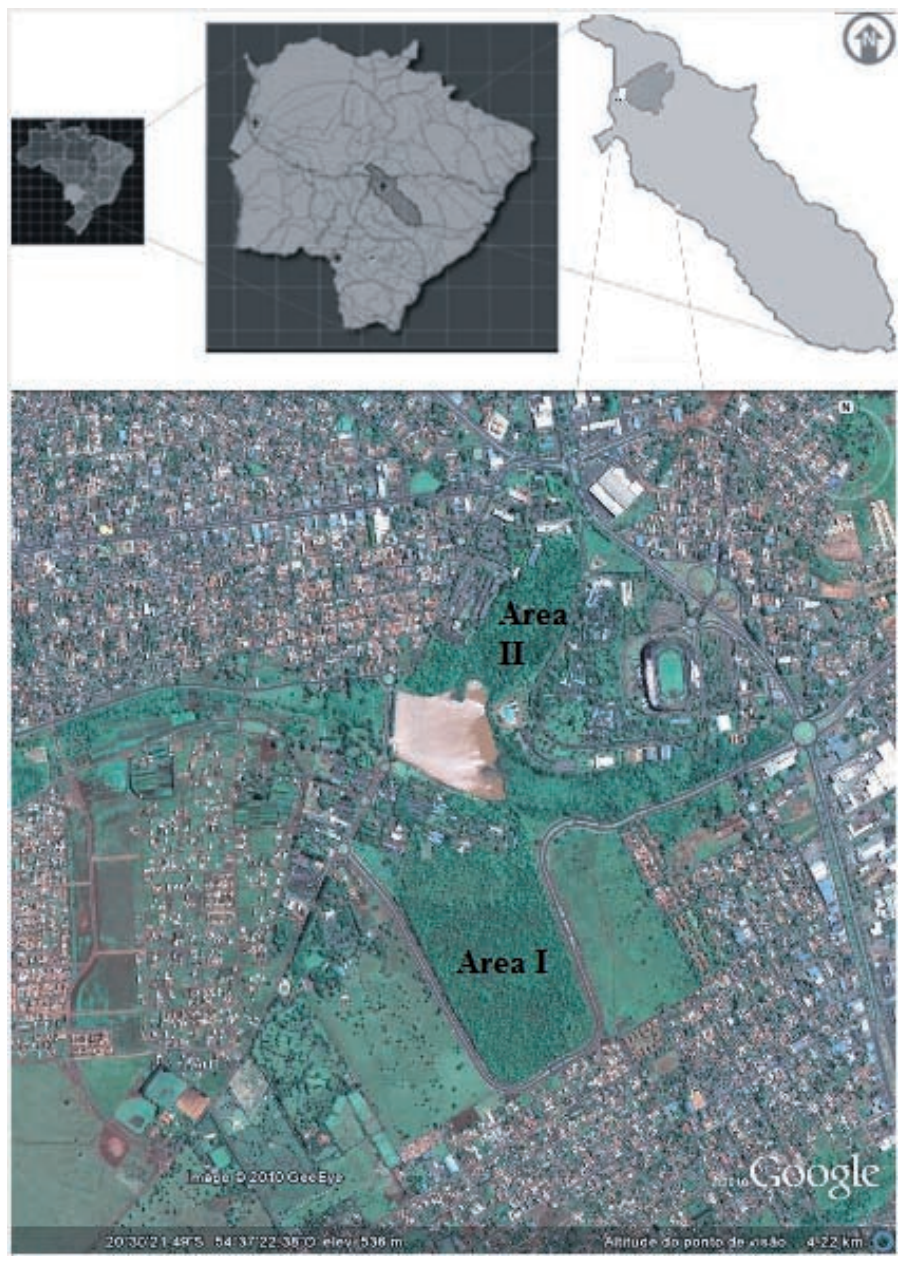

Figura 1. Localização do remanescente contíguo de cerrado pertencente a Reserva Particular do Patrimônio Natural da UFMS (RPPN / UFMS) (Fonte: Google Earth $®$ ).

\section{RESULTADOS E DISCUSSÃO}

Foram registrados 163 indivíduos distribuídos em 62 espécies, seis famílias e 15 subfamílias, para as áreas de estudo pertencentes à RPPN/UFMS em um total de 100 horas-rede de amostragem e 100 horas de armadilhas (Tabela 1).

Em relação à riqueza de espécies, o estudo apresentou uma pequena riqueza de espécies quando comparado a outros estudos 
Tabela 1. Lista de espécies de borboletas registradas de agosto de 2008 a julho de 2009 na RPPN da Universidade Federal de Mato Grosso do Sul, Campo Grande.

Família

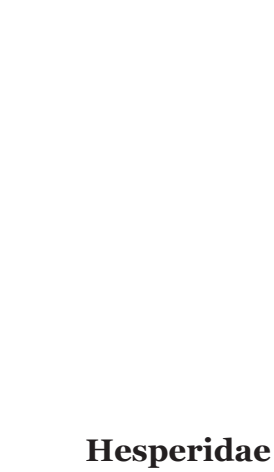

Lycaenidae

sp.1

\begin{tabular}{|ll}
\hline Hesperiinae & $\begin{array}{l}\text { Polites vibex catilina (Plötz) } \\
\text { Pompeius pompeius (Latreille) } \\
\text { sp.2 }\end{array}$ \\
& \\
\hline Pyrginae/Eudamini & Urbanos dorantes dorantes (Stoll) \\
& Urbanus proteus proteus (Linnaeus) \\
& Urbanos simplicius (Stoll) \\
& Urbanus teleus (Hübner) \\
& Urbanus sp. \\
\hline Pyrginae/Pyrgini & \\
\hline & Chiomara sp. \\
& Cogia chalcas (Herrich-Schäffer) \\
& Heliopetes arsalte arsalte (Linnaeus) \\
& Nisoniades sp. \\
& Gorgythion sp. \\
& Grais stigmaticus (Mabille) \\
& Pyrgus orcus (Stoll) \\
\hline
\end{tabular}

Polyommatinae

Hemiargus hanno (Stoll)

Leptotes cassius (Cramer)

Zizula cyna (Edwards)

Theclinae/Eumaeini

Arawacus aetolus (Sulzer)

Arawacus ellida (Hewitson)

Chlorostrymon simaethis (Drury)

Electrostrymon endymion (Fabricius)

Rekoa meton (Cramer)

\begin{tabular}{|c|c|}
\hline Biblidinae/Biblidini & Callicore sorana sorana (Godart) \\
\hline & Eunica bechina (Hewitson) \\
\hline & Hamadryas februa (Hübner) \\
\hline & Mestra hypermestra (Hübner) \\
\hline & Pyrrhogyra neaerea (Linnaeus) \\
\hline & Temenis laothoe meridionalis (Ebert) \\
\hline Charaxinae/Preponini & Archaeoprepona demophon (Linnaeus) \\
\hline Danainae/Danaini & Danaus plexippus erippus (Cramer) \\
\hline Heliconiinae/Acraeini & Actinote pyrrha (Fabricius) \\
\hline & Actinote sp. \\
\hline Heliconiinae/Argynnini & Euptoieta hegesia hegesia (Cramer) \\
\hline Heliconiinae/Heliconiini & Agraulis vanillae maculosa (Stichel) \\
\hline & Dryadula phaetusa (Linnaeus) \\
\hline & Dryas iulia alcionea (Cramer) \\
\hline & Eueides isabella dianasa (Hübner) \\
\hline & Heliconius erato phyllis (Fabricius) \\
\hline Ithomiinae/Godyridini & Hypoleria lavinia (Hewitson) \\
\hline & Hypoleria plisthenes (D'Almeida) \\
\hline Ithomiinae/Mechanitini & Mechanitis polymnia casabranca (Haensch) \\
\hline Ithomiinae/Tithoreini & Tithorea harmonia pseudethra (Butler) \\
\hline
\end{tabular}

Continua.. 
Tabela 1. Continua...

\begin{tabular}{|c|c|c|}
\hline Família & Subfamília/Tribo & Espécie \\
\hline & Morphinae/Morphini & Morpho helenor (Cramer) \\
\hline & Nymphalinae/Kallimini & Anartia amathea roeselia (Eschscholtz) \\
\hline & & Anartia jatrophae (Linnaeus) \\
\hline & & Junonia evarete (Cramer) \\
\hline & & Siproeta traya (Hübner) \\
\hline & Nymphalinae/Melitaeini & Tegosa claudina (Eschscholtz) \\
\hline & & Tegosa similis (Higgins) \\
\hline & Satyrinae/Satyrini & Paryphthimoides phronius (Godart) \\
\hline \multirow{6}{*}{ Pieridae } & Coliadinae & Eurema dina leuce (Boisduval) \\
\hline & & Eurema elathea (Cramer) \\
\hline & & Phoebis argante argante (Fabricius) \\
\hline & & Phoebis philea philea (Linnaeus) \\
\hline & & Pyrisitia nise tenella (Boisduval) \\
\hline & Pierinae/Pierini & Ascia monuste orseis (Godart) \\
\hline \multirow{4}{*}{ Riodinidae } & & sp.3 \\
\hline & Riodininae & Emesis diogenia (Prittwitz) \\
\hline & Riodininae/Nymphidiini & Nymphidium sp. \\
\hline & & Synargis calyce brennus (C. Felder \& R. Felder) \\
\hline
\end{tabular}

realizados em áreas maiores e mais preservadas, como por exemplo, ISERHARD \& ROMANOWSKI (2004), que encontraram em uma área de mata atlântica no Vale do Maquiné, 292 espécies de borboletas em uma área de 4.845 ha ou como Mielke \& CASAGRANDE (1997) que encontraram 426 espécies de borboletas no Parque Estadual do Morro do Diabo que apresenta uma área de 33.845 ha. Esta pequena riqueza em espécies era de se esperar já que, quanto mais próximo de área urbana densa, menor o número de espécies encontradas em fragmentos florestais, fato devido ao desaparecimento de espécies sensíveis à urbanização (RuszcZYK 1999). Já quando comparamos a riqueza de espécies deste trabalho com outros trabalhos realizados em áreas urbanas, a riqueza é quase similar, como por exemplo, SACKIS \& MoRAIS (2008) que registraram 89 espécies de borboletas para o campus da Universidade Federal de Santa Maria (Rio Grande do Sul) ou Silva et al. (2007) que registraram 91 espécies em um fragmento urbano em Minas Gerais.

O número de espécies registradas manteve-se crescente ao longo do estudo, porém a partir da nona coleta (abril de 2009), a inclinação da curva de acumulação mostra-se bastante suave, indicando que o esforço amostral na área atingiu um nível satisfatório. De acordo com a curva de acumulação, não foi possível registrar todas as espécies existentes na área, refletindo a impossibilidade de se amostrar todas as espécies de uma localidade com um número restrito de horas de amostragem (BRown \& Freitas 2000) (Figura 2). Além disso, com um maior tempo de amostragem, o número de espécies registradas tende a aumentar, elevando assim a probabilidade de detectar espécies raras (Summerville et al. 2001) ou até mesmo espécies turistas (GASTON 1996). Os estimadores de riqueza Jack-Knife ( $1^{\circ}$ ordem) e Chao2 mostram respectivamente que entre 81 e $86 \%$ das

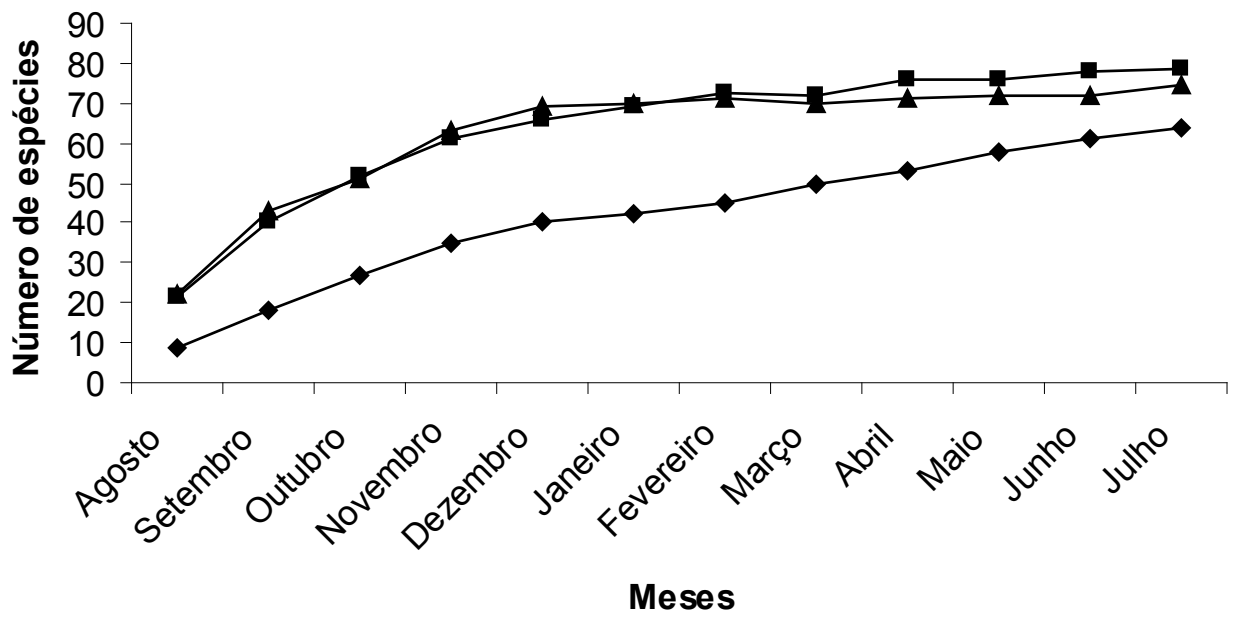

\section{$\longrightarrow$ Observado $\rightarrow-$ Jack-knife $1^{\circ} \longrightarrow$ chao 2}

Figura 2. Curva de acumulação (observado) e estimadores de riqueza (Chao2 e Jack-Knife $1^{\mathrm{a}}$ ordem) de espécies de borboletas encontradas na RPPN da Universidade Federal de Mato Grosso do Sul, Campo Grande, no período de agosto de 2008 a julho de 2009. 
espécies foram registradas durante o período do estudo para a área.

Entre as espécies registradas, 28 pertencem a família Nymphalidae (45,1\%), 16 Hesperiidae (25,8\%), oito Lycaenidae (12,9\%), seis Pieridae (9,7\%) e quatro Riodinidae (6,5\%). A frequência relativa da riqueza de espécies por famílias observadas neste trabalho é similar a de estudos feitos na região Neotropical e no Brasil, sendo Nymphalidae, Hesperiidae e Lycaenidade basicamente as famílias com maior abundância. BECCALONI \& GASTON (1995) demonstram Lycaenidae como a mais rica na região Neotropical seguido por Nymphalidae e Hesperiidae. HEPPNER (1991) demonstra Nymphalidae como a mais rica, seguida de Lycaenidae e Hesperiidae. Em relação ao Brasil, Brown \& Freitas (1999) mostram que as famílias mais ricas em espécies são Lycaenidae, Hesperiidae e Nymphalidae respectivamente. Estudos feitos por MotTA (2002), AoKi \& Sigrist (2006), Pinheiro \& Emery (2006) e Pinheiro et al. (2008), todos compreendidos no Cerrado apresentam pequenas diferenças entre si e entre o presente estudo (Tabela 2). incluem todas as espécies nas listas publicadas ou propostas, em nível Nacional e Estadual, não são encontradas também no presente trabalho, fato devido ao estado de degradação da área, aliado ao seu pequeno tamanho.

Porém apesar de ser muito pequena, estar imersa em uma matriz urbana e ser constantemente impactada, a reserva representa um ambiente importante para a conservação de borboletas dentro do município de Campo Grande. Uma mata urbana pode melhorar de várias maneiras a vida das borboletas que vivem nesta matriz, servindo de habitat para várias espécies, além de contribuir com a diminuição da poluição do ar, aquecimento e variabilidade vegetacional (RuszczYK 1986). Portanto a conservação de matas com vegetação nativa no interior e na periferia da malha urbana seria, assim, uma medida importante para a conservação de uma alta riqueza de borboletas em áreas urbanas (FoRTunAto \& RuszczyK 1997). Pode-se inferir também que a proteção e manejo adequados desta área, poderiam possibilitar um aumento da diversidade de borboletas com o passar do tempo.

Tabela 2. Comparação da riqueza de espécies por família (\%) em estudos feitos na região do Cerrado, com o presente estudo.

\begin{tabular}{lccccc}
\hline \multirow{2}{*}{ Família } & \multicolumn{3}{c}{ Região do Cerrado* } \\
\cline { 2 - 6 } & Motta & P\&E & P et al. & A\&S & RPPN/UFMS \\
\hline Nymphalidae & 44,0 & 32,0 & 65,0 & 30,0 & 45,1 \\
Hesperiidae & 21,0 & 32,5 & 00,0 & 37,5 & 25,8 \\
Lycaenidae & 25,0 & 15,0 & 20,0 & 23,0 & 12,9 \\
Pieridae & 07,0 & 05,0 & 11,0 & 09,5 & 09,7 \\
Riodinidae & 00,0 & 14,0 & 00,0 & 00,0 & 06,5 \\
Papilionidae & 03,0 & 01,5 & 04,0 & 00,0 & 00,0 \\
\hline
\end{tabular}

*Motta = MotтA (2002) em Uberlândia, P\&E = Pinheiro \& Emery (2006), P et al. = Pinheiro et al. $($ 2008) ambos no Distrito Federal, A\&S = Aoki \& Sigrist (2006) no complexo Aporé-Sucuriú, Mato Grosso do Sul e RPPN/UFMS = presente estudo, Campo Grande, Mato Grosso do Sul.

Nymphalidae é registrada por MotTa (2002), AoKi \& Sigrist (2006), Pinheiro \& Emery (2006) e Pinheiro et al. (2008) como uma das famílias mais ricas em espécies, corroborando o presente estudo. Isto acontece porque Nymphalidae talvez seja a família mais diversificada em hábitos e morfologia, sendo responsável por $29 \%$ das espécies de borboletas em comunidades na região Neotropical, como descreve Brown \& FreITAS (1999). Lycaenidae e Hesperiidae também são duas famílias muito abundantes registradas por MotTa (2002), Aoki \& Sigrist (2006), Pinheiro \& Emery (2006) e Pinheiro et al. (2008), sendo estas apresentadas como famílias muito ricas e diversificadas em número de espécies (BROwn \& FreITAS 1999), compondo cerca de 30\% e 40\% respectivamente da fauna de borboletas em qualquer sítio do Neotrópico.

No presente estudo não há registro de nenhuma espécie de Papilionidae, podendo este fato estar relacionado com a área de estudo, já que se trata de um remanescente urbano de Cerrado, antropizado, e que boa parte das espécies desta família são indicadores de ambientes preservados (BROWN \& FrEITAS 1999). Já Pieridae não se mostra muito como uma das famílias mais ricas em espécies neste estudo, mesmo a família Pieridae sendo especialmente abundante em áreas abertas e possuírem muitos representantes comuns em áreas antrópicas (BROwN \& FREITAS 1999).

De acordo com Мотта (2002) as borboletas como: Agraulis vanillae maculosa (Stichel), Heliconius erato phyllis (Fabricius), Junonia evarete (Cramer), Eurema elathea (Cramer) e Ascia monuste orseis (Godart), são comuns, sendo encontradas em muitos habitats e sendo características de locais semi-abertos ou perturbados. Segundo seleção de 103 espécies feitas na Mata Atlântica (ameaçadas, raras) por BRown \& FreITAS (2000), cuja presença indica um ambiente preservado, nenhuma espécie desta lista foi encontrada na área de estudo durante o presente trabalho. As espécies ameaçadas citadas por Brown \& FreITAS (2000) que

\section{AGRADECIMENTOS}

Ao Dr. André Victor Lucci Freitas, Carolina Lemes Nascimento Costa e Poliana Felix Araujo da Universidade Estadual de Campinas pela identificação dos espécimes. Ao Dr. Gustavo Graciolli pelas contribuições e acompanhamento do trabalho.

\section{REFERÊNCIAS}

Almeida, L.M., C.S. Ribeiro-Costa \& L. Marinoni, 2003. Manual de coleta, conservação, montagem e identificação de insetos. Editora Holos, 26p.

Aoki, C. \& M.R. Sigrist, 2006. Inventário dos Visitantes Florais no Complexo Aporé-Sucuriú, p. 145-162. In: Pagotto, T.C.S \& P.R. Souza (Eds.). Biodiversidade do Complexo AporéSucuriú, Subsídios à Conservação e Manejo do Cerrado. Editora UFMS, Campo Grande, 304p.

Beccaloni, G.W. \& K.J. Gaston, 1995. Predicting species richness of Neotropical forest butterflies: Ithomiinae (Lepidoptera: Nymphalidae) as indicators. Biological Conservation, 71: 7786.

Brown, K.S.Jr., 1991. Conservation of Neotropical environments: insects as indicators. p. 349-404. In: Collins, N. M. \& A. Thomas (Eds.). The conservation of insects and their habitats. Academic Press, London, 450 p.

Brown, K.S.Jr. \& A.V.L. Freitas, 1999. Lepidoptera, p. 225-245. In: Brandão, C.R.F. \& E.M. Cancello (Eds.). Biodiversidade do Estado de São Paulo, Brasil. Invertebrados Terrestres. FAPESP, XVI+279p.

Brown, K.S.Jr. \&A.V.L. Freitas, 2000. Diversidade de Lepidoptera em Santa Teresa, Espírito Santo. Boletim do Museu de Biologia Mello Leitão, 11/12: 71-118.

Brown, K.S.Jr. \& O.H.H. Mielke, 1967a. Lepidoptera of the Central Brazil Plateau. I. Preliminary list of Rhopalocera: Introduction, Nymphalidae, Libytheidae. Journal of the Lepidopterists Society, 21: 77-106. 
Brown, K.S.Jr. \& O.H.H. Mielke, 1967b. Lepidoptera of the Central Brazil Plateau. II. Preliminary list of Rhopalocera (continued): Lycaenidae, Pieridae, Papilionidae, Hesperiidae. Journal of the Lepidopterists Society, 21: 145-168.

Camargo, A.J.A. \& V.O. Becker, 1999. Saturniidae (Lepidoptera) from the Brazilian cerrado: Composition and biogeographic relationships. Biotropica, 31: 696-705.

Collier, N., D.A. Mackai, K. Benkendorff, A.D. Austin \& S.M. Carthew, 2006. Butterfly communities in south australian urban reserves: estimating abundance and diversity using the Pollard walk. Austral Ecology, 31: 282-290.

Daily, G.C. \& P.R. Ehrlich, 1995. Preservation of biodiversity in small rainforest patches: rapid evaluations using butterfly trapping. Biodiversity and Conservation, 4: 35-55.

Dessuy, M.B. \& A.B.B. Morais, 2007. Diversidade de borboletas (Lepidoptera, Papilionoidea e Hesperioidea) em fragmentos de Floresta Estacional Decídua em Santa Maria, Rio Grande do Sul, Brasil. Revista Brasileira de Zoologia, 24: 108-120.

Dias, B.F.S., 1996. Cerrados: Uma caracterização. In: Dias, B.F.S. (Ed.). Alternativas de desenvolvimento dos Cerrados: manejo e conservação dos recursos naturais renováveis. Funatura, Brasília, 97p.

Diniz, I.R. \& H.C. Morais, 1995. Larvas de Lepidoptera e suas plantas hospedeiras em um cerrado de Brasília, Distrito Federal, Brasil. Revista Brasileira de Entomologia, 39: 755770.

EMBRAPA - CNPGC, 1985. Boletim Agrometeorológico. Campo Grande, MS.

Emery, E.O., K.S.Jr. Brown \& C.E.G. Pinheiro, 2006. As borboletas (Lepidoptera, Papilionoidea) do Distrito Federal, Brasil. Revista Brasileira de Entomologia, 50: 85-92.

Fahrig, L., 2003. Effects of habitat fragmentation on biodiversity. Annual Review of Ecology, Evolution and Systematics, 34: 487-515.

Fonseca, N.G., A.F. Kumagai \& O.H.H Mielke, 2006. Lepidópteros visitantes florais de Stachytarpheta cayennensis (Rich.) Vahl (Verbenaceae) em remanescente de Mata Atlântica, Minas Gerais, Brasil. Revista Brasileira de Entomologia, 50: 399405.

Fortunato, L. \& A. Ruszczyk, 1997. Comunidades de Lepidópteros frugívoros em áreas verdes urbanas e extra-urbanas de Uberlândia, MG. Revista Brasileira de Biologia, 57: 79-87.

Freitas, A.V.L., R.B. Francini \& K.S. Brown Jr., 2003. Insetos como indicadores ambientais, p. 125-151. In: Cullen, L.Jr., R. Rudran \& C. Valladares-Pádua (Eds.). Métodos de estudo em Biologia da Conservação e Manejo da Vida Silvestre. Fundação Boticário e Editora da UFPR, Curitiba, 652p.

Gaston, K.J., 1996. Species richness: measure and measurement. Apud: Iserhard, C.A. \& H.P. Romanowski, 2004. Lista de espécies de borboletas (Lepidoptera, Papilionoidea e Hesperioidea) da região do vale do rio Maquiné, Rio Grande do Sul, Brasil. Revista Brasileira de Zoologia, 21: 649-662.

Heppner, J.B., 1991. Faunal regions and the diversity of Lepidoptera. Tropical Lepidoptera, 2: 1-85.

Hughes, J.B., C.D. Gretchen \& P.R. Ehrlich, 2000. Conservation of insect diversity: a habitat approach. Conservation Biology, 14: 1788-1797.

Iserhard, C.A. \& H.P. Romanowski, 2004. Lista de espécies de borboletas (Lepidoptera, Papilionoidea e Hesperioidea) da região do vale do rio Maquiné, Rio Grande do Sul, Brasil.
Revista Brasileira de Zoologia, 21: 649-662.

Köppen, W., 1948. Climatologia. Fundo de Cultura Econômica, Buenos Aires.

McAlecee, N., P.J.D. Lambshead, G.L.J. Paterson \& J.G. Gage, 1997. BioDiversity Professional. Beta-Version. The Natural History Museum and The Scottish Association for Marine Sciences.

Mielke, O.H.H. \& M.M. Casagrande, 1997. Papilionoidea e Hesperioidea (Lepidoptera) do Parque Estadual do Morro do Diabo, Teodoro Sampaio, São Paulo, Brasil. Revista Brasileira de Biologia, 14: 967-1001.

Motta, P.C., 2002. Butterflies from the Uberlândia region, central Brazil: species list and biological comments. Brazilian Journal of Biology, 62: 151-163.

Munin, R.L., R.C. Teixeira \& M.R. Sigrist, 2008. Esfingofilia e sistema de reprodução de Bauhinia curvula Benth. (Leguminosae: Caesalpinioideae) em cerrado no CentroOeste brasileiro. Revista Brasileira de Botânica, 31: 15-25.

Pinheiro, C.E.G.\&E.O.Emery, 2006. Thebutterflies (Lepidoptera: Papilionoidea and Hesperioidea) of the Environmental Protection Zone/APA do Gama e Cabeça de Veado, Distrito Federal, Brazil. Biota Neotropica, 6: 1-15.

Pinheiro, C.E.G., I.C. Malinov, T.O. Andrade, J. Maravalhas, M. Moussallem, L.P.A. Deus, L.G.P. Pedrosa \& G. Zanatta, 2008. The butterflies (Lepidoptera, Papilionoidea) of the University Campus Darcy Ribeiro (Distrito Federal, Brasil). Biota Neotropica, 8: 139-144.

Rodrigues, J.J.S., K.S.Jr. Brown \& A. Ruszczyk, 1993. Resources and conservation of neotropical butterflies in urban forests fragments. Biological Conservation, 64: 3-9.

Ruszczyk, A., 1986. Ecologia urbana de borboletas, I. O gradiente de urbanização e a fauna de Porto Alegre RS. Revista Brasileira de Biologia, 46: 675-688.

Ruszczyk, A., 1999. Borboletas: indicadoras da qualidade ambiental. In: Menegat, R. (Ed.). Atlas Ambiental de Porto Alegre. Editora da Universidade, Porto Alegre, 256p.

Sackis, G.D. \& A.B.B. Morais, 2008. Borboletas (Lepidoptera: Hesperioidea e Papilionoidea) do campus da Universidade Federal de Santa Maria, Santa Maria, Rio Grande do Sul. Biota Neotropica, 8: 151-158.

Santos, A.J., 2004. Estimativas de riqueza em espécies. In: Cullen, L.Jr., R. Rudran \& C. Valladares-Padua (Eds.). Métodos de estudos em biologia e manejo da vida silvestre. Editora da UFPR, Curitiba, 665p.

Silva, A.R.M., G.G. Landa \& R.F. Vitalino, 2007. Borboletas (Lepidoptera) de um fragmento de mata urbano em Minas Gerais, Brasil. Lundiana, 8: 137-142.

Summerville, K.S., E.H. Metzler \& T.O. Crist, 2001. Diversity of Lepidoptera in Ohio Forests at local and regional scales: how heterogeneous is the fauna?. Annals of the Entomological Society of America, 94: 583-591.

Wilson, E.O., 1997. Biodiversidade. Nova Fronteira, 70op.

Wood, B. \& M.P. Gillman, 1998. The effects of disturbance on forest butterflies using two methods of sampling in Trinidad. Biodiversity and Conservation, 7: 597-616.

\section{Recebido em: 26/01/2012}

Aceito em: 12/04/2012

\section{Como citar este artigo:}

Bogiani, P.A., R. Aranda \& C.O.F. Machado, 2012. Riqueza de Borboletas (Lepidoptera) em um Fragmento Urbano de Cerrado em Mato Grosso do Sul, Brasil. EntomoBrasilis, 5(2): 93-98.

Acessível em: http://www.periodico.ebras.bio.br/ojs/index.php/ebras/article/view/204
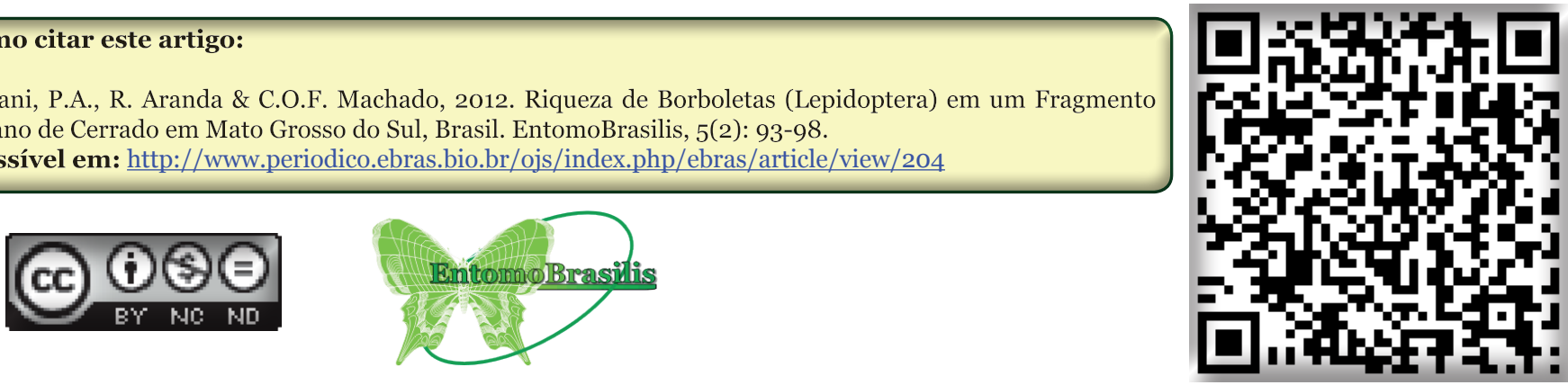